\title{
Malformaciones congénitas. Incidencia en la raza mapuche
}

En la medida que un país se desarrolla en sus aspectos socioeconómicos, se produce un cambio en las causas determinantes de morbimortali$\mathrm{dad}^{1-\dot{b}-6-11}$. Es así como los problemas derivados de causas genéticas o degenerativas emergen y desplazan a las etiologías infecciosas o nut ritivas.

* Pediatra, Servicio Pediatria Hospital Regional de Temuco.

**Pediatra, jefe Lnidad Recién Nacidos y Prematuros. Hospital Regional de Temuco

***Profesor de Bioestadística, Depto. Medicina Humana, Universidad de Chile, Sede Temuco.

*** Matronas de la Unidad de Recién Nacidos, Hospi. tal Regional de Temuco

*****Cirujano Plástico, Hospital Regional de Temuco.
Dres. Guillermo Soza C. * Dra. Lidia Benitez M..** María Ibaceta L. ; ${ }^{* * * *}$ Sr. René Peña F.;*** Sras. Mercedes Garcia.*** María Teresa Torres. ${ }^{* * *}$ y Carmen Morales $* * *$

En la infancia, el impacto de las malformaciones congénitas (MC) como determinante o telón de fondo en la patología de esta edad es enorme.

Se sabe que un $20 \%$ de las muertes que se producen en el último trimestre del embarazo y un $15 \%$ de las producidas en el período neonatal son productos de $\mathrm{MC}^{7 \cdot{ }^{12}}$. En la primera infancia, esta influencia se hace imprecisable si se consideran las malformaciones internas o funcionales, errores congénitos del metabolismo ${ }^{10}$.

Al hablar de MC se incluyen cuadros chinicos de etiología diferente. Hoy se sabe que serian cuatro los mecanismos productores básicos: 1. Mutaciones genéticas. 
2. Alteraciones cromosómicas.

3. Factores del macro o microambiente (noxa intrauterina).

4. Multifactorial (genético ambiental).

Como se puede apreciar, el papel genético es fundamental en la etiología de las MC. La variable étnica o raza es un factor que ha merecido numerosos estudios.

En la literatura revisada, no hemos encontrado referencias a estudios de MC en la raza mapuche.

En recientes publicaciones ${ }^{6-11}$ se menciona la necesidad de contar con estadisticas regionales dadas las variaciones étnicas de nuestro país.

Nuestro propósito al efectuar esta investigación, fue determinar si había diferencias en las MC de los recién nacidos mapuches en relación a un grupo considerado no mapuche, en cuanto a incidencia y tipo de malformación. Paralelamente medir la influencia de algunas variables ambientales y su eventual prevención.

\section{MATERIAL Y METODO}

El estudio fue realizado en la Maternidad y Servicio de Recién Nacidos ( $R N$ ) del Hospital Regional de Temuco.

El tiempo de observación fue desde octubre de 1972 a septiembre de 1973 .

Durante ese período se examinaron 4.748 recién nacidos vivos. De este universo, se detectaron $76 \mathrm{RN}$ con anomalías congénitas pesquisables externamente.

Se consideró RN mapuche aquel cuyos progenitores tuviesen ambos apellidos de este origen (válido para investigaciones de este tipo). El resto se agrupó como no mapuche.

En ambos casos se determinaron los siguientes antecedentes:

1. Tipo de malformación.

2. Sexo RN. Peso. Edad gestacional.

3. Residencia de los padres, consanguinidad. Legitimidad de la unión. Paridad. Abortos previos.

4. Patología en la madre. Antecedentes de ingestión de medicamentos o cualquier noxafísica. Alcoholismo. Tabaquismo.

Recolección de datos en sistema de tarjetas precodificadas.

\section{RESULTADOS}

En la tabla 1 se aprecia el número de RN vivos en ambas razas. El menor número de origen mapuche es explicado por la mayor ruralidad de este grupo poblacional con baja atención de parto hospitalario.

Tabla 1

$N^{\circ}$ DE RECIEN NACHDOS VIVOS SEGLN RAZAS.

MATERNIDAD HOSPITAL REGIONAL DE.

TEMUCO. OCTURRE 1972 A SEPTIEMBRE 1973

\begin{tabular}{lrr}
\hline Razas & \multicolumn{1}{c}{ R.N. } & $\%$ \\
\hline Mapuches & 592 & 12,7 \\
No mapuches & 4.156 & 87,3 \\
\cline { 2 - 3 }$\quad$ Total & 4.748 & 100,0 \\
\hline
\end{tabular}

En la tabla 2 se observa el número de malformaciones según razas y tasas por mil RN vivos. Destaca inmediatamente el mayor número de malformados en la raza mapuche:

$$
\left(p_{1}-p_{2}=10.6\right) \text {. }
$$

Considerando similitud y comparabilidad en los grupos, dados que la selección es aleatoria; madres concurren espontáneamente al hospital, mujeres en edad fértil, en un ambiente geográfico común, cabe preguntarse si la diferencia observada es debida al azar o si tiene valor estadístico. Con un nivel de significación de 5\% previamente establecido, encontramos que existe sólo $2,7 \%$ de probabilidad que la diferencia se deba al azar, luego es estadísticamente significativa. En el comentario se analizará este hallazgo

\section{Tabla 2}

MALFORMACIONES CONGENITAS SEGUN RAZAS. TASAS POR 1.000 RECIEN NACIDOS VIVOS. MATERNIDAD HOSPITAL DE TEMLCO. OCTUBRE 1972 A SEPTIEMBRE 1973

\begin{tabular}{lrrr}
\hline \multicolumn{1}{c}{ Razas } & R.N. & \multicolumn{2}{c}{$\begin{array}{l}\text { Malformaciones } \\
\text { No tasa } \times 1.000\end{array}$} \\
\hline Mapuches & 592 & 15 & 25,3 \\
No mapuches & 4.156 & 61 & 14,7 \\
\cline { 2 - 4 } Total & 4.748 & 76 & 16,0 \\
\hline
\end{tabular}


Las tasas para población no mapuche y para el total de malformaciones son cercanas $y$ significativamente coincidentes con resultados de investigaciones preliminares ${ }^{6}$.

En la tabla 3 se analizan los tipos de malformaciones encontradas y por razas con el porcentaje para cada grupo según la Clasificación Internacional de Enfermedades.
Con la finalidad de someter a análisis comparativo los datos, se confeccionó tabla 4 refundiendo malformaciones por sistemas y expresando el resultado en tasas por mil $\mathrm{RN}$ vivos $\mathrm{y}$ por razas.

En el grupo no mapuche la primera causa corresponde al rubro esqueléticas, muy cerca le siguen las MC de oido, cara y cuello, síndrome

\section{Taba 3}

TIPOS DE MALFORMACIONES CONGENITAS OBSERVADAS EN 74 RECIEN NACIDOS VIVOS SEGUN RAZAS MAPUCHE Y NO MAPUCHE. HOSPITAL REGIONAL TEMUCO Octubre 1972 a septiembre 1973

\begin{tabular}{|c|c|c|c|c|c|}
\hline \multirow{2}{*}{ Código* } & \multirow{2}{*}{ Malformaciones } & \multicolumn{2}{|c|}{ Mopuches } & \multicolumn{2}{|c|}{ No mapuches } \\
\hline & & $N^{\infty}$ & $\%$ & $\lambda^{\infty}$ & $\%$ \\
\hline \multirow[t]{4}{*}{$740-743$} & Sistema Nervioso & - & - & 7 & 11,5 \\
\hline & Acrania & - & - & 4 & \\
\hline & Hidrocefalia & -- & - & 2 & \\
\hline & Espina bífida & - & - & 1 & \\
\hline 744 & Ojo & - & - & - & $\ldots$ \\
\hline \multirow[t]{5}{*}{745} & Oido, cara, cuello & 6 & 40,0 & 15 & 24,6 \\
\hline & Papiloma preauricular & 4 & - & 7 & \\
\hline & Atrofia pab. auricular + & 1 & & & \\
\hline & Inperforación del oido & 1 & & 6 & \\
\hline & Malform. pab. auricular & & & 2 & \\
\hline \multirow[t]{2}{*}{$746-747$} & Cardiovascular & - & & 1 & 1,6 \\
\hline & Dextrocardia + C. I. V. & - & & 1 & \\
\hline 748 & Respiratorio & - & - & - & - \\
\hline \multirow[t]{4}{*}{749} & Labio lepormo $y / 0$ palatar hendido & 6 & 40,0 & 6 & 9,8 \\
\hline & Fisura palatina aislada & - & & 2 & \\
\hline & Labio leporino simple & 1 & & 1 & \\
\hline & Labio leporino c/fisura & 5 & & 3 & \\
\hline \multirow[t]{3}{*}{$750-751$} & Gastrointestmal & - & - & 3 & 4,9 \\
\hline & Atresia esofágica & - & & 1 & \\
\hline & Imperforación anal con fistula & - & & 2 & \\
\hline \multirow[t]{2}{*}{$752-753$} & Genitourinano & - & - & 2 & 3,3 \\
\hline & Hipospadia & 一 & & 2 & \\
\hline \multirow[t]{6}{*}{$754-756$} & Esquelético & 2 & 13,3 & 16 & 26,2 \\
\hline & Pie bot & 1 & & 7 & \\
\hline & Polidactilia extrem. inferiores & 1 & & 4 & \\
\hline & Sindactilia & - & & 1 & \\
\hline & Displasia cadera & - & & 1 & \\
\hline & Amputación congénita dedos de la mano & - & & 3 & \\
\hline 757 & Dermatológico & - & - & - & - \\
\hline \multirow[t]{3}{*}{758} & Otras & - & & 2 & 3.3 \\
\hline & Angioma (227) & - & & 1 & \\
\hline & Rudimentos denatrios (520.6) & - & & 1 & \\
\hline \multirow[t]{3}{*}{759} & Sindrome & 1 & 6,7 & 9 & 14,8 \\
\hline & Síndrome de Down & 1 & & 9 & \\
\hline & Total & 15 & 100,0 & 61 & $1(10), 0$ \\
\hline
\end{tabular}

*Codigo: clasificación internacional de enfermedades. Rev. 1965 oms. 
Tabla 4

CLASIFICACION RESUMIDA Y TASAS POR 1.000 R. N. VIVOS SEGUN RAZAS

\begin{tabular}{|c|c|c|c|}
\hline \multirow{2}{*}{ Código } & \multirow{2}{*}{ Malfarmaciones } & \multicolumn{2}{|c|}{ Tasa $\times 1000 R$. N. vivos } \\
\hline & & Mapuches & No mapuches \\
\hline $740-743$ & Sistema Nervioso & 一 & 1,7 \\
\hline 744 & Ojo & - & - \\
\hline 745 & Ódo, cara, cuello & 10,1 & 3,6 \\
\hline $746-747$ & Cardiovascular & - & 0,2 \\
\hline 748 & Respiratorio & - & $\cdots$ \\
\hline 749 & Labio leporino y/o paladar hendido & 10,1 & 1,4 \\
\hline $750-751$ & Gastmintestinal & - & 0,7 \\
\hline $752-753$ & Genitourinario & - & 0,5 \\
\hline $754-756$ & Esquelético & 3,4 & 3.9 \\
\hline 757 & Dermatológico & & \\
\hline 758 & Otras & & 0,5 \\
\hline 759 & Sindrome & 1,7 & 2,2 \\
\hline Total & & 23,3 & 14,7 \\
\hline
\end{tabular}

de Down, tercer lugar y Sistema Nervioso el cuarto, quinto lugar labio leporino y paladar hendido. En estadísticas de recientes presentaciones de anomalías en la Región Metropolitana ${ }^{6} 11$ hay semejantes frecuencias con mayor tasa para las esqueléticas, determinada fundamentalmente por la displasia de cadera, que en nuestra casuística aparece con sólo un caso.

El sindrome de Down ocupa también el tercer lugar en el trabajo aludido.

En el grupo mapuche hay notables diferencias y algunos hechos llamativos. El primer lugar está determinado por el grupo de oído, cara y cuello en igual porcentaje que labio leporino y paladar hendido, seguido por esqueléticas y síndrome de Down.

El $40 \%$ de las malformaciones de mapuches está determinado por anomalías de oído, cara y cuello. Otro $40 \%$ corresponde a labio leporino y/o paladar hendido. En el grupo no mapuche el porcentaje para oído, cara y cuello es de $24,6 \%$ y en labio leporino $9,8 \%$. En publicación de referencia los porcentajes son de 22 y $7 \%$, respectivamente, más parecidas a la frecuencia de estas malformaciones en la población considerada no mapuche.

Las tablas 5,6 y 7 resumen los puntos con-

Tabla 5

CARACTERISTICAS DE MALFORMADOS SEGUN RAZA HOSPITAL TEMUCO

Octubre 1972 - Septiembre 1973

\begin{tabular}{|c|c|c|c|c|c|c|c|c|c|c|c|c|c|c|}
\hline \multirow[b]{2}{*}{$R a z a$} & \multicolumn{2}{|c|}{ Residencia } & \multirow{2}{*}{ Total } & \multicolumn{2}{|c|}{ sexpr } & \multirow{2}{*}{ Total } & \multicolumn{3}{|c|}{ Peso } & \multirow{2}{*}{ Total } & \multicolumn{3}{|c|}{ Edad gestacional } & \multirow{2}{*}{ Total } \\
\hline & Urbano & Rural & & $M$ & $F$ & & $\begin{array}{l}1000 \\
2000\end{array}$ & $\begin{array}{r}2001 \\
2500\end{array}$ & $\begin{array}{l}-2501 \\
\text { ó más }\end{array}$ & & $\begin{array}{l}\text { Prelier } \\
\text { mino }\end{array}$ & $\begin{array}{l}\text { érm } \\
\text { no }\end{array}$ & $\begin{array}{l}\text { Postér } \\
\text { mino }\end{array}$ & \\
\hline Mapuche & 5 & 10 & 15 & 9 & 6 & 15 & & 3 & 12 & 15 & 3 & 12 & - . & 15 \\
\hline No mapuche & 39 & 22 & 61 & 30 & 31 & 61 & 5 & $\therefore$ & 49 & 61 & 10 & 51. & $\bar{\cdots}$ & 61 \\
\hline Total & 44 & 32 & 76 & 39 & 37 & 76 & 5 & 10 & 51 & 76 & 13 & 63 & & 76 \\
\hline
\end{tabular}


Tabla 6

ALGUNAS VARIABLES DE MALFORMADOS SEGUN RAZAS. HOSPITAL TEMUCO, 1972-73

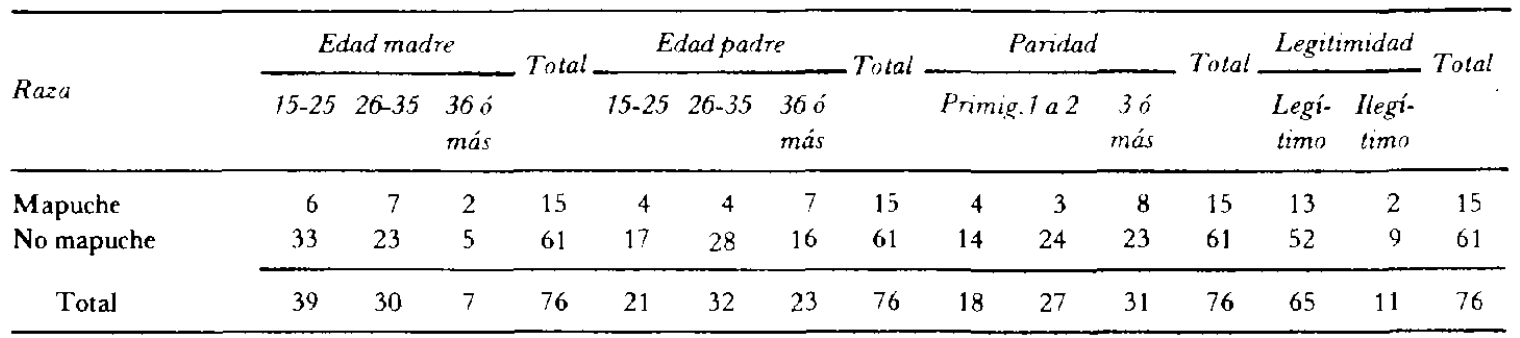

Tabla 7

ALGUNOS ANTECEDENTES MALFORMADOS SEGUN RAZAS HOSPITAL TEMUCO, 1972-73

\begin{tabular}{|c|c|c|c|c|c|c|c|c|c|c|c|c|}
\hline \multirow[t]{2}{*}{$R a z a$} & \multicolumn{2}{|c|}{ Patolog. Embarazo } & \multirow{2}{*}{ Total } & \multicolumn{2}{|c|}{$\begin{array}{c}\text { Embarazo } \\
\text { Drogas }\end{array}$} & \multirow[t]{2}{*}{ Total } & \multicolumn{2}{|c|}{$\begin{array}{c}\text { Malform. } \\
\text { Hiyos Anter. }\end{array}$} & \multirow[t]{2}{*}{ Total } & \multicolumn{2}{|c|}{$\begin{array}{l}\text { Malform. } \\
\text { Familar }\end{array}$} & \multirow{2}{*}{ Total } \\
\hline & $S i$ & No & & Sí & No & & $S_{i}^{\prime}$ & No & & $S_{i}$ & No & \\
\hline Mapuche & 4 & 11 & 15 & 5 & 10 & 15 & 3 & 12 & 15 & 1 & 14 & 15 \\
\hline No mapuche & 19 & 42 & 61 & 19 & 42 & 61 & 4 & 57 & 61 & 4 & 57 & 61 \\
\hline Total & 23 & 53 & 76 & 24 & 52 & 76 & 7 & 69 & 76 & 5 & 71 & 76 \\
\hline
\end{tabular}

signados en la pauta de observación, siempre para ambos grupos raciales.

Sólo un tercio de los RN mapuches son de origen urbano. No hay diferencias significativas en cuanto al sexto del malformado.

Existe un mayor porcentaje de prematurez $(20 \%)$ que para la población general. Este hecho ha sido comentado por otros autores ${ }^{6-8-10-11}$ relacionando el rubro edad materna, nutrición en el "ambiente" que rodea a un malformado. Sin embargo, la mayor parte de las madres fue menor de 35 años y los padres sobre los 26 años. Predominaron las madres multiparas ${ }^{8}$.
Se constató patología previa o concomitante al embarazo en 1 de 3 madres, la que se distribuye en la siguiente forma: enfermedad respiratoria (7), toxemia (7), tuberculosis (2), diabetes aguda (1), epilepsia (1), apendicectomía (2), colecistectomía (1), dispositivo intrauterino, anticonceptivos, y embarazo (1).

Droga y embarazo en $30 \%$ de las madres (vitaminas, hormonas, diuréticos, salicílicas, anticonvulsivante, antiparasitario, abortivos).

El dato de malformaciones en hijos anteriores o familiares se dio con más frecuencia en los RN mapuches (20\%). Corresponde a 2 casos de labio leporino en hijos anteriores, anomalia 
semejante a los casos índice y un caso de polidactilia que también se presentó en el caso observado.

Este antecedente se detectó en el $10 \%$ de no mapuches y corresponde a diferentes anomalias. Polidactilia la más constante similar al caso índice, variando el resto (cardiopatía, displasia, sordomudez congénita, labio leporino).

Se buscó correlación entre las malformaciones más frecuentes y los datos consignados en la pauta. Por su bajo número, no permitió establecer diferencias significativas. En este intento destacó:

La mayor parte de malformaciones esqueléticas (especialmente pie bot) y de oído, cara y cuello se presentan en madres sobre 30 años con más de 3 hijos.

En estos dos tipos de MC se dio con más frecuencia $(50 \%)$ el antecedente de drogas durante el embarazo.

En cambio llamó la atención que la mayor parte de madres de $\mathrm{RN}$ con síndrome de Down estaba bajo los 30 años.

\section{DISCUSION}

La mayor incidencia de malformaciones en la raza mapuche, en relación al grupo no mapuche, estuvo determinada fundamentalmente por las anomalías de oído, cara y cuello, y labio leporino y/o paladar hendido. Este fue el hallazgo más sobresaliente en esta investigación. Probablemente con estudios más prolongados en el tiempo con mayor número de malformados, esta tendencia pueda ser aclarada.

Esta diferencia en la expresión fenotípica del rasgo anómalo se correlaciona con la variable étnica, hecho ya evidenciado en diferentes grupos raciales de Singapur (Searle, 1958).

Cabe preguntarse hasta qué punto la condición raza no se acompaña de un habitat o microambiente que influya a través del tiempo en la facilidad expresiva de locus genéticos alterados, quizás por fenómenos nutritivos o de endogamia propio de culturas que resisten su integración a influencias de otras culturas, fenómeno notable en el mapuche. Esta interacción genético-ambiental es materia de investigación para especialistas. Nos limitamos a describir el hecho.

De mayor importancia, porque ya han sido descritos, son las relaciones de las MC detectadas con el peso del niño, paridad de la madre y antecedente "conocido" de drogas o patología en el embarazo.

Este hecho, aunque es conocido, se debe denunciar con mayor insistencia por cuanto es uno de los pocos factores prevenibles en cuya acción importante papel corresponde al personal médico.

Muchos alcances y conclusiones podrán obtenerse a través de investigaciones posteriores. Creemos haber dado una pequeña luz a un apasionante tema.

\section{RESUMEN}

Estudio prospectivo de incidencia y tipo de malformaciones en dos grupos étnicos: mapuches y no mapuches con relación a algunos antecedentes ambientales.

Trabajo realizado en el Servicio de Recién Nacidos del Hospital Regional de Temuco durante el periodo transcurrido entre octubre de 1972 y septiembre de 1973 . Se estudiaron 76 malformaciones.

Lo más llamativo fue la mayor tasa de malformaciones en la raza mapuche a expensas del primer arco branquial y digestiva alta (labio leporino, fisura).

Se analiza y discute la interacción genético-ambiental

\section{REFERENCIAS}

- Barral, I. La Genética Humana y la Salud Pública. Tercer Seminario de Salud Matema e Infantil, 1972.

2 Year Book of Pediatrics : 37-8, 1975

3 Organización Mundial de la Salud. Clasificación Internacional de Enfermedades, 1965

4 Dobzhansky, T. Proced. W.P. Conference, Belgrado, 1965. Reseña de los Principios Básicos de la Genética.

5 Meneghello, J. Pediatria. Intermédica, 1972

6 Vizzer, J. y otros. Pediatría 18:37-41, 1975.

Velson, W. Tratado de Pediatría. pp. 336-9. Barcelonà, 1974.

B Peyser, J. y otros. Rev. Chil. Pediat, fI(8):628.32, 1969.

- Smith, D.W. Atlas de Malformaciones Somáticas en el Niño. Barcelona, Edit. Pediátrica, 1972.

10 Stevenssm, A.C. Frecuencia de las enfermedades congénitas y hereditarias, con especial referencia a las mutaciones. Curso Latino-Americano de Salud Materna e Infantil, 1970 (Apartado)

11 Valenzuela, V.C. y otros. Pediatría $18(2): 114-7$ 1975 .

12 Holmes, L.B. New Engl. J. of Med. 291(15):763-773, Oct. 1974 